\title{
Recovery from severe dysphagia in systemic sclerosis - myositis overlap: a case report.
}

\author{
Keith J Chinniah, Girish M Mody
}

Department of Rheumatology, School of Clinical Medicine, College of Health Sciences, University of KwaZulu-Natal and Inkosi Albert Luthuli Central Hospital, Durban, South Africa

\begin{abstract}
Background: Dysphagia is common in inflammatory myopathies and usually responds to corticosteroids. Severe dysphagia requiring feeding by percutaneous endoscopic gastrostomy is associated with significant morbidity and high mortality.

Clinical case: A 56-year old African Black woman initially presented with systemic sclerosis (SSC) - myositis overlap and interstitial lung disease. She responded to high dose corticosteroids and cyclophosphamide followed by azathioprine, with improvement in her lung function and regression of the skin changes. Six years later she had a myositis flare with severe dysphagia. Her myositis improved after high doses of corticosteroids, azathioprine and two doses of intravenous immunoglobulin (IVIG). As her dysphagia persisted, she was fed via a percutaneous endoscopic gastrostomy (PEG) tube and given a course of rituximab. Her dysphagia gradually resolved and the PEG tube was removed within two months. She received another dose of rituximab six months later and continued low dose prednisone and azathioprine. Her muscle power improved, weight returned to normal and she remained well 20 months after hospital discharge.

Conclusion: Our patient with SSC-myositis overlap and severe dysphagia requiring PEG feeding, improved with high dose corticosteroids, azathioprine, two courses of IVIG and rituximab, and remained in remission 20 months after hospital discharge. Keywords: Severe dysphagia, systemic sclerosis, myositis overlap.

DOI: https://dx.doi.org/10.4314/ahs.v17i2.38

Cite as: Chinniah KJ, Mody GM. Recovery from severe dysphagia in systemic sclerosis - myositis overlap: a case report. Afri Health Sci. 2017;17(2): 593-596. https://dx.doi.org/10.4314/abs.v17i2.38
\end{abstract}

\section{Introduction}

Dysphagia is reported in up to $90 \%$ of patients with systemic sclerosis (SSC) and $20-84 \%$ of inflammatory myopathies, and may occur at any stage during the course of the disease ${ }^{1}$. Patients may experience nasal regurgitation, coughing while swallowing and dysphagia for solids and liquids ${ }^{2}$. As a result, percutaneous endoscopic gastrostomy (PEG) feeding is required to prevent aspiration pneumonia.

\section{Corresponding author: \\ Girish M Mody, \\ Department of Rheumatology, \\ Room 528, School of Clinical Medicine, \\ University of KwaZulu-Natal, \\ 719 Umbilo Road, Durban, South Africa, 4001 \\ Fax Number: 0027312604482 \\ Telephone: 0027312604284 \\ E-mail:modyg@ukzn.ac.za}

The initial treatment of inflammatory myopathies is high dose corticosteroids. Patients' refractory to corticosteroids or who require high doses of corticosteroids as maintenance therapy, may respond to immunosuppressive agents such as methotrexate, azathioprine, cyclophosphamide, cyclosporine or mycophenolate mofetil ${ }^{3}$. Intravenous immunoglobulin (IVIG) and rituximab are effective in patients with severe, progressive or refractory inflammatory myopathy ${ }^{4-8}$. Patients with refractory oesophageal involvement require PEG feeding and 82.2\% of patients may improve with repeated courses of $\mathrm{IVIG}^{4}$.

We report about a patient with SSC - myositis overlap who was admitted with a myositis flare and severe dysphagia. She showed a gradual response to feeding via a nasogastric tube followed by PEG tube feeding and intensive immunosuppressive therapy. Signed informed consent for publication was obtained from the patient and permission was also obtained from the Biomedical Research Ethics Committee of the University of KwaZulu-Natal.

\section{Case report}

A 56-year old African Black woman was referred to the African Health Sciences Vol 17 Issue 2, June, 2017 
rheumatology department at Inkosi Albert Luthuli Central Hospital in Durban, South Africa in June 2008. She had SSC for one year with involvement of the face, chest, upper and lower limbs, and interstitial lung disease. She also had an inflammatory myopathy with weakness of the neck flexors and proximal muscles of the upper and lower limbs. The anti-nuclear antibody was positive in a low titre of 1: 40 with a speckled and nucleolar pattern. The test for extractable nuclear antigens was negative. The creatine kinase $(\mathrm{CK})$ was 260 units per litre $(\mathrm{U} / \mathrm{L}$ ) (normal 20 - $180 \mathrm{U} / \mathrm{L}$ ). Electromyography was consistent with a myopathic process and muscle biopsy showed atrophic, degenerative and regenerative muscle fibres, mild fibrosis and sparse peri-vascular lymphocytes and plasma cells. She had a severe restrictive defect on pulmonary function tests and high resolution computed tomography (HRCT) of the chest showed intra- and interlobular septal thickening, traction bronchiectasis, basal pleural thickening and ground glass opacification consistent with active interstitial lung disease.

A diagnosis of SSC myositis overlap with interstitial lung disease was made and she received 2 weekly $500 \mathrm{mg}$ cyclophosphamide pulses for twelve weeks followed by azathioprine $100 \mathrm{mg} /$ day. Her initial prednisone dose of 30 $\mathrm{mg} /$ day was gradually tapered to $5 \mathrm{mg} /$ day. A response of the interstitial lung disease was noted with increase in the lung volumes on pulmonary function tests. Her muscle power and functional status also improved.

She remained well on azathioprine (75-100 $\mathrm{mg}$ per day) and $5 \mathrm{mg}$ prednisone. There was gradual regression of the skin changes on clinical assessment. During the latter part of 2012, she had a myositis flare (CK 5085 U/L) which responded to a temporary increase in corticosteroids. In May 2013 Mycobacterium tuberculosis was identified on sputum culture and she was treated with a fixed dose combination of isoniazid, rifampicin, pyrazinamide and ethambutol for two months followed by isoniazid and rifampicin for four months.

In February 2014, she was admitted with a history of progressive increase in muscle weakness and severe dysphagia. She had nasal speech and severe muscle weakness involving the neck flexors and proximal muscles in the upper and lower limbs. Her weight was $43 \mathrm{Kg}$ and the CK was raised to $6173 \mathrm{U} / \mathrm{L}$. She was treated with three daily pulses of $500 \mathrm{mg}$ methylprednisolone followed by oral prednisone $50 \mathrm{mg} /$ day and azathioprine $150 \mathrm{mg} /$ day. She was fed via a nasogastric tube and required frequent suctioning as she was unable to swallow her saliva. A flexible naso-endoscopy noted pooling of saliva in both pyriform fossae. As there was no significant improvement, she was given a course of IVIG a week later. During February 2014, she also had recurrent episodes of supraventricular tachycardia which were treated acutely with adenosine and then controlled with atenolol.

There was gradual improvement in her peripheral muscle power (CK $2178 \mathrm{U} / \mathrm{L}$ ) but she still had severe dysphagia. She was given a further three day course of 250 mg methylprednisolone pulses about four weeks after the initial dose. Her muscle power improved further with a reduction in the $\mathrm{CK}$ to $1262 \mathrm{U} / \mathrm{L}$. The dysphagia persisted and a second course of IVIG was given in March 2014. In April 2014, the CK had fallen to $438 \mathrm{U} / \mathrm{L}$ and she developed a Klebsiellsa Pneumoniae urinary tract infection followed by a severe pneumonia with hypoxia which responded to broad spectrum antibiotics. A follow up naso-endoscopy was unchanged and therefore a PEG tube was inserted and she was started on rituximab $500 \mathrm{mg}$ weekly based on her body surface area. She developed sepsis around the site of the PEG tube after three weekly doses of rituximab and therefore the fourth dose was omitted.

She subsequently showed a gradual and progressive improvement in her muscle power and dysphagia. In June 2014, the CK returned to normal (73 U/L), she was ambulant with good muscle power and she was able to swallow normally. The PEG tube was removed and she was discharged on prednisone $10 \mathrm{mg}$ per day and azathioprine $150 \mathrm{mg} /$ day.

Her muscle power improved and weight increased during regular out-patient follow-up. In November 2014, the CK rose to $470 \mathrm{U} / \mathrm{L}$ in the absence of dysphagia or weakness. In view of the severity of her previous illness and increased risk of infection, we chose to give her a lower dose of rituximab and she received two further $500 \mathrm{mg}$ doses. The second dose was delayed by two weeks due to a urinary tract infection and diarrhea. Her CK fell to $91 \mathrm{U} / \mathrm{L}$ and she continued azathioprine $100 \mathrm{mg} /$ day and prednisone $5 \mathrm{mg} /$ day. 
She remained well during 2015 and her CK at the time of her last visit in February 2016 was 115 U/L. Her muscle power was good and she was eating normally. Her weight increased to $61.9 \mathrm{~kg}$. Repeat blood tests showed that the ANF, PM-Scl, Jo-1, Mi-2, SSA and SSB antibodies were negative.

\section{Discussion}

Dysphagia is common in patients with inflammatory myopathies and its management includes rehabilitation measures, corticosteroids and other immunosuppressive agents, and rarely surgical measures such as cricopharyngeal myotomies and dilatations ${ }^{9}$. Although the majority of patients with dysphagia respond to corticosteroids, case reports and small case series provide evidence for the efficacy of immunosuppressive agents such as azathioprine, methotrexate, cyclosporine, cyclophosphamide and MMF for severe dysphagia ${ }^{3}$. A Cochrane database review failed to detect any high quality randomized controlled trials to support the efficacy of any immunosuppressive medication (other than prednisone) in polymyositis $(\mathrm{PM})$ or dermatomyositis $(\mathrm{DM})^{10}$. Rituximab has been shown to be effective for refractory myositis in separate series of 30 French and 13 Dutch patients ${ }^{6,7}$. Oddis et al showed improvement in $83 \%$ of 202 juveniles and adults with inflammatory myositis treated with rituximab in a randomized study ${ }^{8}$. However, there are no reports of its use for severe dysphagia.

Marie et al reported a French multicenter series of 73 patients with PM and DM who received IVIG for steroid refractory oesophageal involvement ${ }^{4}$. Nine (12.3\%) patients required feeding via a PEG or percutaneous endoscopic jejunostomy. They found a favorable early response in $82.2 \%$, and therefore recommended high dose corticosteroids and IVIG as initial therapy for severe dysphagia. Oh et al reported a series of 62 patients with inflammatory myopathies and dysphagia and noted a high mortality in seven $(64 \%)$ of their 11 patients who required a $\mathrm{PEG}^{9}$. Das et al reported three patients with SSC- myositis overlap with severe dysphagia who required PEG feeding for prolonged periods ranging from 5-20 months ${ }^{11}$.

There are several interesting features in our patient. She had diffuse SSC- myositis overlap with regression of skin changes after treatment with cyclophosphamide for her interstitial lung disease as noted in 13 (11.1\%) of $117 \mathrm{pa}-$ tients with SSC who were treated with cyclophosphamide for interstitial lung disease ${ }^{12}$. Although the myositis in our patient responded to two courses of pulse methylprednisolone, high doses of oral corticosteroids, azathioprine and two doses of IVIG, rituximab was given as she had persistent dysphagia and three episodes of infection on high doses of steroids. The recovery of the dysphagia with removal of the PEG within 6 weeks of rituximab therapy permitted discharge of the patient from hospital. The contribution of the rituximab is uncertain as her recovery may be due to the delayed effect of corticosteroid and IVIG therapy. The management of patients with severe dysphagia in resource constrained settings is challenging. Fortunately, our patient has maintained her weight and normal function 20 months after hospital discharge.

\section{Acknowledgement}

We thank Mr. Fundile Habana for assistance with the preparation of this manuscript.

We acknowledge the financial support from the Aaron Beare Family Chair of Rheumatology Endowment Fund (for a research assistant).

\section{Disclosure statement}

Professor Girish M Mody served as a member of the Vimovo advisory board for AstraZeneca South Africa and is a member of the Global Advisory Board for GSK Consumer Healthcare's Pain Franchise.

\section{Conflict of interest}

Dr. Keith J Chinniah reports no conflict of interest.

Indexing terms: Rituximab Polymyositis

Dysphagia Intravenous immunoglobulin

Systemic sclerosis Percutaneous endoscopic gastrostomy (PEG)

Nasoendoscopy

\section{References}

1. Sheehan N. Dysphagia and other manifestations of oesophageal involvement in the musculoskeletal diseases. Rheumatology. 2008; 47(6): 746-52.

2. Ebert EC. Review article: the gastrointestinal complications of myositis. Aliment Pharmacol Ther. 2010; 31(3): 359-65. 
3. Dalakas MC. Inflammatory muscle diseases. N Engl J Med. 2015; 372(18): 1734-47.

4. Marie I, Menard JF, Hatron PY, et al. Intravenous immunoglobulins for steroid-refractory esophageal involvement related to polymyositis and dermatomyositis: a series of 73 patients. Arthritis Care Res (Hoboken). 2010; 62(12): 1748-55.

5. Wang DX, Shu XM, Tian XL, et al. Intravenous immunoglobulin therapy in adult patients with polymyositis/ dermatomyositis: a systematic literature review. Clin Rheumatol. 2012; 31(5): 801-6.

6. Couderc M, Gottenberg JE, Mariette X, et al. Efficacy and safety of rituximab in the treatment of refractory inflammatory myopathies in adults: results from the AIR registry. Rheumatology (Oxford). 2011; 50(12): 2283-9.

7. Mahler EA, Blom M, Voermans NC, van Engelen BG, van Riel PL and Vonk MC. Rituximab treatment in patients with refractory inflammatory myopathies. Rheumatology (Oxford). 2011; 50(12): 2206-13.

8. Oddis CV, Reed AM, Aggarwal R, et al. Rituximab in the treatment of refractory adult and juvenile dermatomyositis and adult polymyositis: A randomized, placebo-phase trial. Arthritis \& Rheumatism. 2013; 65(2): 314-24. 9. Oh TH, Brumfield KA, Hoskin TL, Stolp KA, Murray JA and Bassford JR. Dysphagia in inflammatory myopathy: clinical characteristics, treatment strategies, and outcome in 62 patients. Mayo Clin Proc. 2007; 82(4): 441-7. 10. Gordon PA, Winer JB, Hoogendijk JE and Choy EH. Immunosuppressant and immunomodulatory treatment for dermatomyositis and polymyositis. Cochrane Database Syst Rev. 2012; 8: Cd003643.

11. Das L, Bowden A, Cooper RG, Mitchell W, O'Sullivan $\mathrm{M}$ and Herrick AL. Percutaneous endoscopic gastrostomy feeding--a life-saving intervention in ssc-myositis overlap with pharyngeal dysfunction. Rheumatology (Oxford). 2012; 51(8): 1518-20.

12. Foocharoen C, Mahakkanukrauh A, Suwannaroj S and Nanagara R. Spontaneous skin regression and predictors of skin regression in Thai scleroderma patients. Clin Rheumatol. 2011; 30(9): 1235-40. 